\title{
Assessment of a wireless headband for automatic sleep scoring
}

\author{
H. Griessenberger • D. P. J. Heib • A. B. Kunz • \\ K. Hoedlmoser $\cdot$ M. Schabus
}

Received: 16 December 2011 /Revised: 20 June 2012 / Accepted: 17 July 2012 /Published online: 21 September 2012

(C) The Author(s) 2012. This article is published with open access at Springerlink.com

\begin{abstract}
Purpose Classically, professional assessment of sleep is done in the sleep laboratory using whole-night polysomnography (PSG). However, given a misbalance between accredited sleep laboratories and the large amount of patients suffering from sleep disorders, only few receive appropriate diagnostic assessment. Recently, some lowcost home sleep scoring systems have been proposed, yet such systems are rarely tested scientifically. The aim of the present study was to evaluate the staging accuracy of the home sleep scoring system Zeo (Newton, MA, USA).

Methods A final sample of 21 nights from ten subjects (aged 23-45) was digitally recorded with PSG as well as with the Zeo system. We compared scorings of Zeo (on an epoch-be-epoch basis) with the Somnolyzer $24 \times 7$ (an automatic staging algorithm), expert scorers as well as the freeware SleepExplorer.
\end{abstract}

Electronic supplementary material The online version of this article (doi:10.1007/s11325-012-0757-4) contains supplementary material, which is available to authorized users.

H. Griessenberger · D. P. J. Heib · K. Hoedlmoser · M. Schabus Department of Psychology, Laboratory for Sleep, Cognition and Consciousness Research, University of Salzburg,

Hellbrunnerstrasse 34,

5020 Salzburg, Austria

\section{A. B. Kunz}

Christian Doppler Clinic, Department of Neurology,

Paracelsus Private Medical University,

Ignaz-Harrerstraße 79,

5020 Salzburg, Austria

\section{Schabus $(\bowtie)$}

Laboratory for Sleep, Cognition and Consciousness Research,

Department of Psychology, University of Salzburg,

Hellbrunnerstrasse 34,

5020 Salzburg, Austria

e-mail: manuel.schabus@sbg.ac.at
Results It was revealed that Zeo shows moderate overall agreement as compared to our study standard Somnolyzer $24 \times 7(\kappa=0.56)$. The most obvious performance difference between Zeo and both other scoring approaches was stage wake (sleep onset latency+wake after sleep onset). While Zeo detected only $40.8 \%$ of the study standard wake epochs, $70.1 \%$ were detected by the expert scorers and $83.4 \%$ by the SleepExplorer, respectively.

Conclusions Data suggest that the Zeo system produces acceptable sleep scoring for stage REM, light and deep sleep, with a specific weakness in correctly detecting waking periods.

Keywords Sleep staging $\cdot$ Wireless headband $\cdot$ EEG $\cdot$ Sleep disturbance

\section{Introduction}

According to the most recent criteria of the American Academy of Sleep Medicine [1], at least three electroencephalogram (EEG) channels, two unipolar electrooculogram (EOG) and three electromyogram (EMG) channels are needed for appropriate measurement of sleep stages. This standard polysomnographic setup requires expensive equipment as well as trained personnel and can therefore only be performed in professional sleep laboratories. The main advantage of a laboratory-based sleep assessment is the on-site presence of health care professionals checking for various sleep disorders as well as the control for environmental variables. Sleeping in a laboratory setting however is unusual for subjects and can therefore result in altered sleep architecture, such as present in the well-known first night effect (e.g. [2, 3]). Furthermore, the high financial costs, the low availability of sleep laboratories and the long waiting periods strongly restrict the number of consecutive nights in the sleep laboratory. Unfortunately, many sleep disorders do 
not occur on a daily basis, and it is therefore likely that disorders are missed if only one to two nights can be assessed. Hence, other technologies have been developed and tested for home sleep applicability ([4-6], for review see [7]). These alternative devices are systems specialized for detection of certain sleep disorders (e.g. obstructive sleep apnea $[8,9])$. On the other hand, a software for automatic sleep scoring (e.g. ASEEGA system [10]) or detection of sleep/wake states by radiofrequency biomotion sensors [11] provides reliable alternatives to classic polysomnography.

In the present study, a wireless home sleep monitoring system was evaluated for a mixed group of (subclinical) insomnia patients and good sleeper controls. The study aim was an objective external evaluation, which discusses weaknesses and strengths of the Zeo system. We leave it open to the reader to conclude the applicability to his or her field of interest. Specifically, we studied the Zeo system (Axon Laboratories; Newton, MA, USA) $[12,13]$ which scores the night in 30-s epochs and four stages (wake, light sleep, deep sleep and rapid eye movement sleep) simply using three dry frontal electrodes. Data are compared with our study standard Somnolyzer $24 \times 7[14,15]$. Additionally, we report the comparison scorings between our study standard and both an expert scorer (A.K.) and the automatic sleep analysis of the freeware SleepExplorer (El Ratón Networks). Therefore, we tested the Zeo system against an automatic (SleepExplorer), semiautomatic (Somnolyzer) and manual sleep staging.

\section{Methods}

Data were obtained from a larger study cohort where people suffering from primary insomnia were compared to good sleeper controls (age range $=20-57$ ). All participants were requested to be non-habitual smokers (less than five cigarettes a day). A preceding entrance examination included Diagnosis of Psychiatric Disorders according to DSM IV (Structured Clinical Interview for DSM Disorders) and psychometric tests (e.g. personality questionnaires). Patients (primary insomnia) were classified according to the research diagnostic criteria of Edinger and colleagues [16]. Healthy controls were regular sleepers which was determined by clinical interviews and sleep quality questionnaires (Pittsburgh Sleep Quality Index score $<5$ ). Originally, 37 records (nights) of 13 subjects were included in this study. Due to technical difficulties (reoccurring data loss over the night in the Zeo data recordings), only 21 recordings could be analyzed. The remaining records had to be excluded as synchronization between polysomnographic EEG data and Zeo data was not possible in an epoch-by-epoch manner. Finally, each recording consisted of a minimum of $6 \mathrm{~h}$ (720 epochs) of undisturbed sleep stages. Participants provided a written informed consent. The electroencephalogram (EEG) was recorded utilizing Synamps EEG amplifiers (NeuroScan Inc., El Paso, Texas). All signals were filtered $(0.10 \mathrm{~Hz}$ high-pass filter; $70 \mathrm{~Hz}$ low-pass filter; $50 \mathrm{~Hz}$ notch filter) and digitized online with $500 \mathrm{~Hz}$ sampling rate. Twentythree EEG channels (Fp1, Fpz, Fp2, F7, F3, Fz, F4, F8, T3, C3, Cz, C4, T4, T5, P3, Pz, P4, T6, O1, Oz, O2, as well as A1 and $\mathrm{A} 2$ for later re-referencing), four electrooculogram (EOG) channels, one bipolar submental electromyogram (EMG) channel, one bipolar electrocardiogram (ECG) channel and one bipolar respiratory channel (chest wall movements) were placed. Electrodes were attached according to the international electrode (10-20) placement system (cf. [17]).

All recordings were scored by four different systems: (1) the semiautomatic scoring of Somnolyzer $24 \times 7$ (serving as our study standard [14]), (2) the manual scoring of one expert scorer (A.K.) (according to AASM rules [1]), (3) the automatic pre-scoring of the freeware software SleepExplorer (El Ratón networks, http://www.sleepexplorer.de/) and (4) the Zeo device (instrument of interest). Expert scorers were blind to the outcome of the other utilized methods.

We decided to use the semi-automatic Somnolyzer $24 \times 7$ as our study standard due to the reported reliable sleep classification which is also manually reviewed and revised if needed. Moreover, it is to be noted that reliability scores were shown to be better between Somnolyzer $24 \times 7$ and manual scorers than the reliability between human scorers [14] (also see Supplementary Table 1).

The Zeo headband consisted of three frontal wireless dry electrodes on a lightweight headband which was placed at the forehead-below electrodes Fp1, Fpz, Fp2. Like defined by classical staging criteria, the Zeo system scores a night in 30 -s epochs but only in four stages (wake, light sleep, deep sleep and rapid eye movement sleep). Yet note that the underlying ZEO scoring algorithms are unfortunately proprietary and not open for in-depth evaluation.

Epoch-by-epoch comparison

For each recording, raw data were synchronized so that the onset of the first epoch was identical for all scoring systems. Epoch synchronization was also corrected for EEG data loss in case of bathroom visits or electrode adjustments during the night. The four different scoring systems were tested for their agreement on the following four stages: [wake (sleep onset latency (SOL)+ wake after sleep onset (WASO)), light sleep (N1 or N2), deep sleep (N3) and rapid eye movement sleep (REM)].

\section{Statistical analyses}

Statistical analyses were performed using PASW 18.0.0 software (SPSS Inc., Chicago, IL). The significance level was set to $p<0.05$. The outcome of Somnolyzer $24 \times 7$ 
stagings (defined as study standard) was compared against the other three approaches with a focus on the Zeo system.

Epoch by epoch agreement was defined as the percentage of epochs that were assigned the same stage. Paired sample $t$ tests were calculated for percentage agreements to the study standard for overall agreement, stages wake, light sleep, deep sleep and rapid-eye-movement sleep. We also calculated sensitivity and positive predictive values (PPV).

Cohen's kappa $(\kappa)$ was used to assess the agreement for pairwise comparisons. Partial correlation coefficients, controlling for the total number of epochs, were used for quantitative analysis. Bland-Altman plots served for visualizing agreement between two given scoring approaches. Comparisons were done for our study standard versus Zeo, expert scorer and SleepExplorer analysis.

\section{Results}

The mean age of the final study sample was 32.5 years $(\mathrm{SD}=7.63$; range, 23-45). Three of the subjects reported no sleep disorder (in total six recordings), whereas seven subjects were suffering from primary insomnia.

According to our study standard, time spent in bed was $470.07 \mathrm{~min}(\mathrm{SD}=15.76 \mathrm{~min})$, total sleep time was $435.14 \mathrm{~min}(\mathrm{SD}=29.48 \mathrm{~min})$ and sleep efficiency was $92.5 \%(\mathrm{SD}=4.48 \%)$. On average, the subjects spent $85.98 \mathrm{~min}(\mathrm{SD}=30.83 \mathrm{~min})$ in deep sleep (N3), $256.74 \mathrm{~min}(\mathrm{SD}=45.76 \mathrm{~min})$ in light sleep $(\mathrm{N} 1+\mathrm{N} 2)$ and $92.43 \mathrm{~min}(\mathrm{SD}=27.09 \mathrm{~min})$ in REM sleep. SOL was $10.79 \mathrm{~min}(\mathrm{SD}=11.18 \mathrm{~min})$, and WASO was $24.14 \mathrm{~min}$ $(\mathrm{SD}=12.1 \mathrm{~min})$. Table 1 illustrates the percent agreement

Table 1 Percent agreement table for epoch labeling between Zeo and comparison scorings (Somnolyzer 24×7, Expert and SleepExplorer)

\begin{tabular}{llrrrr}
\hline \multirow{5}{*}{ Zeo } & & & \\
\cline { 3 - 6 } Somnolyzer & Wake & 40.83 & 26.79 & 29.24 & 3.14 \\
& REM & 11.54 & 73.69 & 13.40 & 1.37 \\
& Light & 7.21 & 9.17 & 80.04 & 3.59 \\
& Deep & 1.30 & 0.08 & 36.48 & 62.13 \\
Expert scorer & Wake & 40.05 & 24.03 & 30.96 & 4.96 \\
& REM & 13.94 & 70.65 & 14.28 & 1.13 \\
& Light & 4.88 & 9.46 & 79.13 & 6.52 \\
& Deep & 0.48 & 0.67 & 29.94 & 68.91 \\
SleepExplorer & Wake & 31.39 & 30.93 & 34.01 & 3.67 \\
& REM & 13.39 & 67.77 & 18.00 & 0.83 \\
& Light & 2.89 & 4.62 & 84.93 & 7.56 \\
& Deep & 0.86 & 0.49 & 29.45 & 69.20 \\
\hline
\end{tabular}

of the Zeo system with all other methods across the 21 recorded nights (for a contingency table based on each epoch, see Supplemental Table 1).

\section{Stage comparisons}

The overall agreement (all 19,738 epochs from all subjects) was $72.6 \%(\kappa=0.56)$ between Somnolyzer $24 \times 7$ and Zeo, $80.9 \%(\kappa=0.69)$ between Somnolyzer $24 \times 7$ and expert scorer and $74.2 \%(\kappa=0.61)$ between Somnolyzer $24 \times 7$ and SleepExplorer. Paired sample $t$ tests revealed a significant difference between the Zeo and expert scorer agreements $(t=-4.048 ; p<.01)$ indicating a significant higher agreement score for the expert scorer with the study standard. Similar differences were revealed between the expert and SleepExplorer agreements $(t=5.204 ; p<.01)$ indicating better overall agreements with the study standard for the expert scorer.

All PPV and scores for sensitivity are reported in Table 2. Zeo revealed the worst sensitivity values in stage wake (40.8\%) indicating that less than half of the study standard wake epochs are correctly detected. Best scoring agreement was represented by expert scorer in light sleep (86.4\%).

\section{Bland and Altman plots}

Figure 1 shows the Bland-Altman plots visualizing the agreements of Somnolyzer $24 \times 7$ and the three other scoring instruments for wake parameters (SOL+WASO). There are no systematic under- or overestimation of the Zeo scorings (see Supplemental material). The greatest mean underestimation (21 $\mathrm{min}$ ) of Zeo as compared to the study standard is revealed for deep sleep, which reaches significance $\left(t_{20}=\right.$

Table 2 Classification results between Somnolyzer $24 \times 7$ and comparison scorings (Zeo, Expert and SleepExplorer)

\begin{tabular}{lclcccc}
\hline & & & Wake & REM & Light & Deep \\
\hline $\begin{array}{lcllll}\text { Zeo } \\
\text { \% OA }\end{array}$ & 72.6 & Sensitivity & 40.8 & 73.7 & 80.0 & 62.1 \\
Cohen's kappa & 0.56 & PPV & 32.0 & 67.4 & 79.2 & 82.2 \\
Expert & & & & & & \\
\% OA & 80.9 & Sensitivity & 70.1 & 80.1 & 86.4 & 69.8 \\
Cohen's kappa & 0.69 & PPV & 53.1 & 81.6 & 82.4 & 93.7 \\
SleepExplorer & & & & & & \\
\% OA & 74.2 & Sensitivity & 83.4 & 74.2 & 75.3 & 67.2 \\
Cohen's kappa & 0.61 & PPV & 37.7 & 70.2 & 83.4 & 91.3 \\
\hline
\end{tabular}

$\% O A$ percent overall agreement, sensitivity percentage of actual sleep state epochs that are labelled as sleep state epochs by classifier, $P P V$ positive predictive value (percentage of epochs labelled as sleep state that are correctly labelled) 
Fig. 1 Bland-Altman plots of sleep parameters SOL and WASO showing differences between Somnolyzer $24 \times 7$ and a Zeo, b expert c SleepExplorer scorings. The $x$-axes indicate the average from both the study standard and comparison scoring of wake after sleep onset and sleep onset latency. The difference is expressed as the comparison score minus the study standard score. The mean difference and the limits of agreement ( $\pm 1.96 \mathrm{SD})$ are represented as dashed lines. Note that high variabilities are dominant for Zeo scorings a

$\stackrel{\circ}{\circ}$

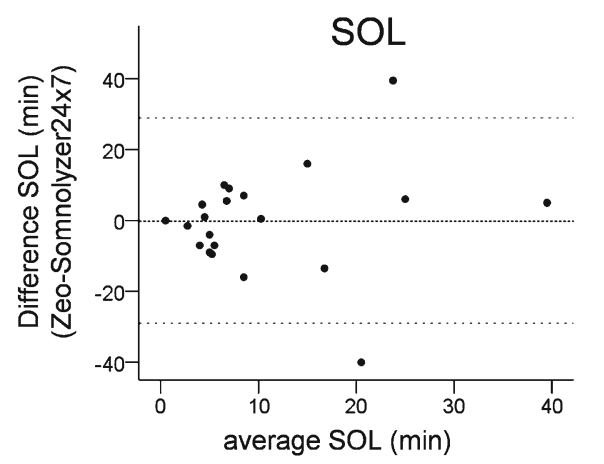

b

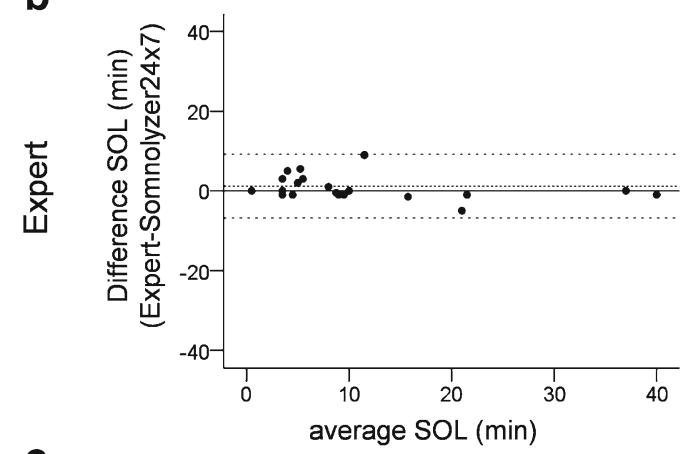

C

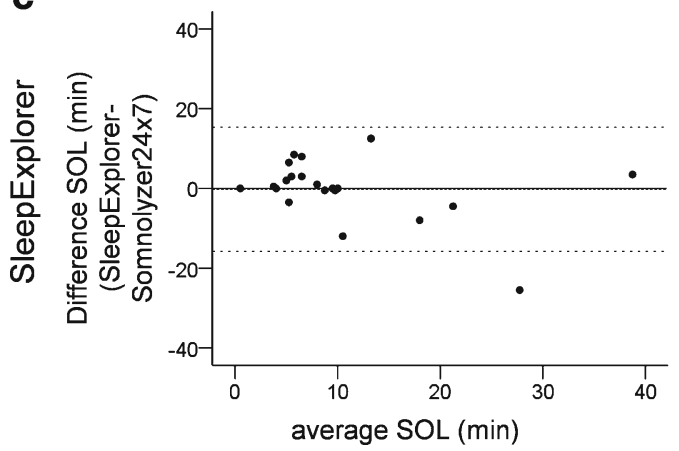

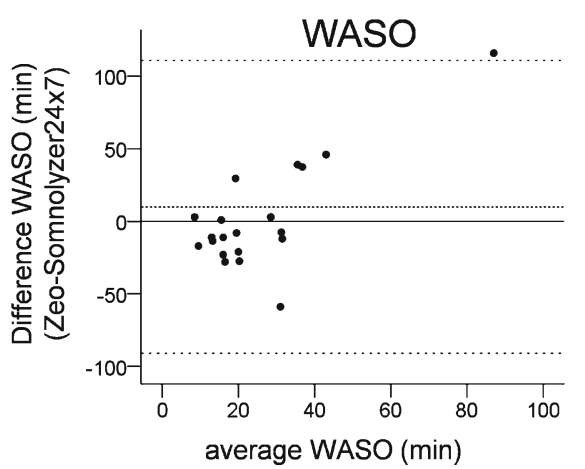
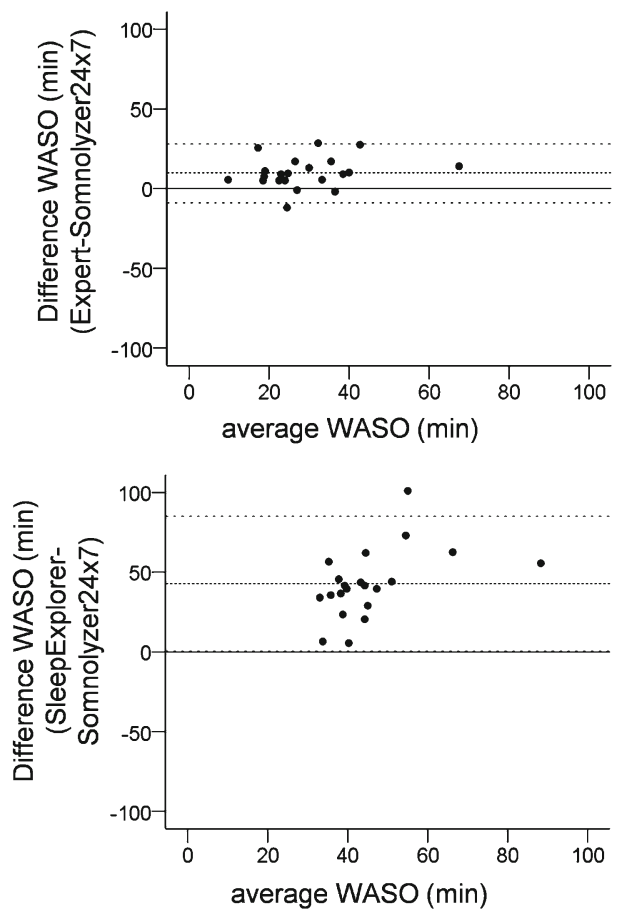

4.225; $p<0.001)$. As shown in Fig. 1a, high variability for waking stages, that is in SOL and WASO, is evident. Yet, note that epoch-by-epoch agreement is better reflected in Table 1.

\section{Correlations}

Correlations between Somnolyzer $24 \times 7$ and Zeo stage minutes revealed significant results for light sleep $(r=.480$, $p<0.05)$ and deep sleep $(r=.695, p<0.01)$. SOL, WASO and REM sleep were not significantly related. For a better overview, Fig. 2 depicts this mismatch. Significant correlations between expert scorer and Somnolyzer $24 \times 7$ were found in light sleep $(r=.680, p<0.01)$, deep sleep $(r=.856, p<0.01)$, SOL $(r=.951, p<0.01)$ and WASO $(r=.634, p<0.01)$. The automatic sleep analysis software SleepExplorer showed significant study standard correlations in SOL $(r=.714, p<$ $0.01)$, light sleep $(r=.561, p<0.05)$, deep sleep $(r=.693, p<$ $0.01)$ and REM sleep $(r=.633, p<0.05)$.

\section{Discussion}

The results of this evaluation study show that the Zeo system provides a moderate sleep scoring with an overall agreement of $72.7 \%$. A Kappa coefficient of 0.56 indicates a reasonable agreement according to Landis and Koch [18]. Zeo revealed the worst sensitivity in stage wake $(40.8 \%$ epoch-by-epoch agreement; cf. Table 1), a mean underestimation of deep sleep of $21 \mathrm{~min}$ and additionally low correlation coefficients for SOL and WASO (cf. Fig. 1). Best agreement was revealed for light sleep (80\%).

In comparison to Zeo, the overall agreement of the expert scorer was $80.9 \%$, and interestingly, even the freely available automatic analysis tool SleepExplorer revealed an agreement of $74.2 \%$. The worst sensitivity for expert scorer and sleep explorer tool was stage deep sleep (69.81\%; $67.23 \%$ ). It has to be fairly taken into account that the latter two scorings rely on full PSGs, whereas the Zeo system has to derive its values from three dry, prefrontal electrodes. 

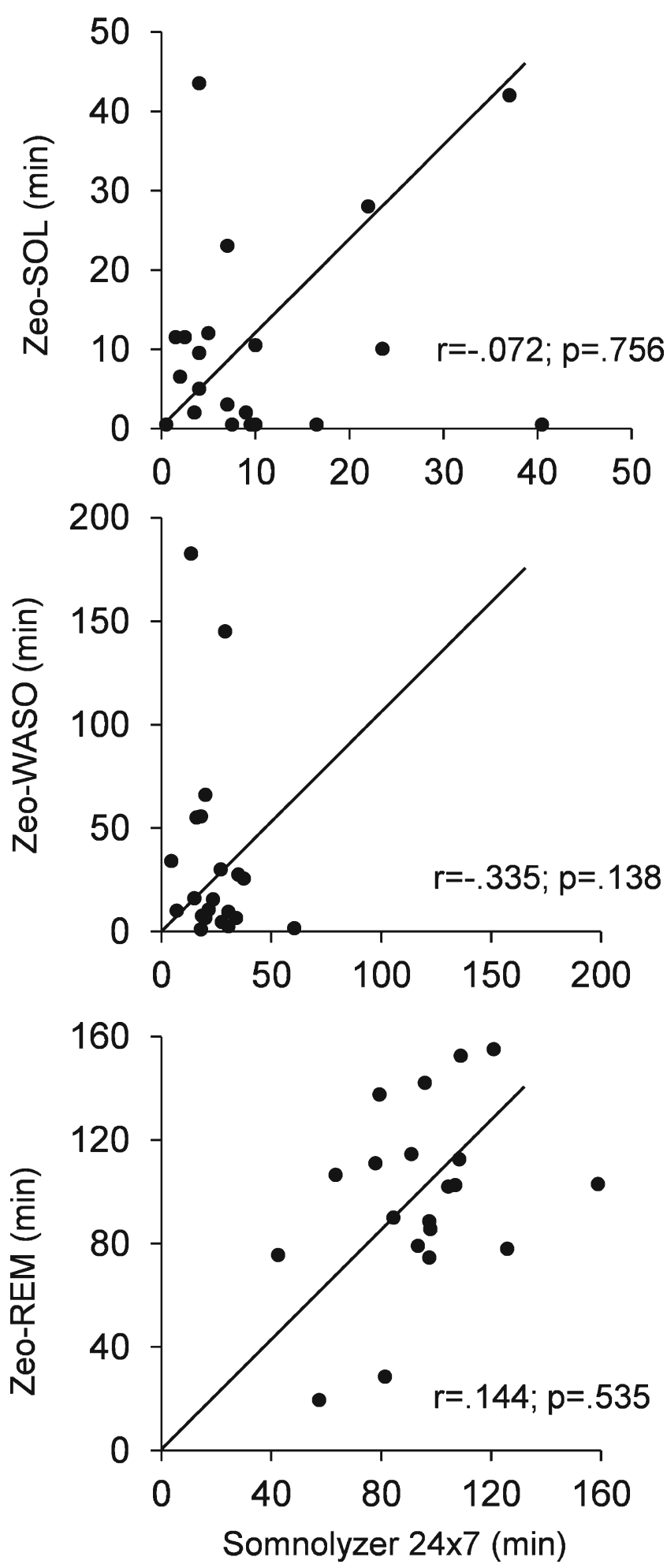

Fig. 2 Scatter plots depicting the agreement of Somnolyzer $24 \times 7$ with Zeo for SOL, WASO and REM (top to bottom). Note that the SOL agreement for Zeo and study standard shows high deviations. Dots are scattered around the $45^{\circ}$ line of identity

To put the results in context, the wireless sleep assessment system Zeo appears to perform similar to other home sleep monitoring systems. Existing actigraphy systems provide epoch-by-epoch accuracies for sleep-wake separation of 75-85\% [19]. Devices which use just a single EEG channel show an accuracy of $84.9 \%$ (i.e. [10]). Earlier reported in-house Zeo investigation revealed epoch-perepoch accuracies between 73 and $91 \%$ [12, 13]. Moreover, the agreement of stage wake was $64 \%$ in these studies as compared to our findings of $40.8 \%$. This difference might also be due to our more inhomogeneous testing sample (healthy as well as primary insomnia patients). On the other hand, sleep architecture proved to be indistinguishable between the two groups, which is also related to the fact that primary insomnia is often found to be a subjective rather than objective complaint [20].

Although the Zeo results can be viewed as acceptable given the ultra quick and easy handling of the device, it is necessary to consider that only about half of all recorded nights could be used for statistical analyses. The rest of the data had too many missing values, mainly because of losing the headband during the night. On the other hand, one can certainly argue that there is no reason for not recording a week or more of data with the Zeo until a reliable set of data has been acquired. Specifically, it is evident that the Zeo system shows weak detection rates for parameters SOL and WASO and as a consequence of stage wake. As these values are often key features when assessing sleep quality, it is necessary to be specifically cautious in interpreting these Zeo measures. Due to the fact that Zeo does not systematically under- or overestimate waking, it is difficult to interpret provided SOL and WASO values.

A potential issue of our study is the unequal number of analyzed nights from patients and healthy volunteers. Yet, we believe that just a mixed real-world sample of that kind is ideal for testing sleep scoring systems: what is still missing is a fullblown evaluation study of home-based systems such as the Zeo together with ambulatory PSG. One big advantage of home-based systems might be the assessment of more habitual sleep at home, especially in groups of subjects which are hard to study otherwise (elderly and children).

One other limitation of this study may be the fact that at present we only tested PSG nights of healthy sleepers as well as primary insomnia patients. It is important to check if results would differ more if patients with sleep disorders other than insomnia are included (e.g. periodic limb movement disorders, restless legs syndrome, parasomnia and sleep apnea). The present study cannot directly address these questions and has to leave this evaluation open to future studies. Just for the integration of multiple channel recordings (EOG, EMG and EEG) expert knowledge seems to be most solid. We also expect that automatic classifiers might have serious problems when it comes to bad PSG data quality, whereas expert scorers might still be able to extract the critical sleep features to assess sleep reliably. Yet this point has to be addressed in the future and is open to discussion. 
In summary, moderate sensitivity and positive predictive value scores were revealed for the home-based Zeo system with stage waking showing the biggest deviance from our study standard. Therefore, systems like Zeo might have a promising future if they can overcome limitations such as data loss during the night or insufficient SOL and WASO detection. Yet, due to the weak detection rates for SOL and WASO, systems of that kind cannot be suggested for home-based diagnostic of sleep disorders to date. Further improvements are needed in order to allow that practical devices such as the Zeo system can be recommended complimentary to sleep diaries and even to assist the clinical decision process for a variety of sleep disorders. Objective evaluation and independent studies of various groups are however inevitable if these systems intend to be approved by scientific standards.

People simply interested in getting a rough picture of their sleep over weeks might already benefit today by utilizing inexpensive sleep scoring devices such as the Zeo system. Manufacturers, however, should communicate limitations more readily and provide information regarding possible shortcomings such as insufficient wake detection or sleep onset latency.

Any tool to enhance awareness of sleep hygiene would greatly benefit our health in a society in which workplace demands and chronic stress induce unhealthy sleep habits. Today such reliable home-based sleep scoring systems are still awaited.

Acknowledgments This research was supported by the Austrian Science Fund (FWF: P-21154-B18).

Conflict of interest The authors declare that they have no conflict of interest.

Open Access This article is distributed under the terms of the Creative Commons Attribution License which permits any use, distribution, and reproduction in any medium, provided the original author(s) and the source are credited.

\section{References}

1. Iber C, American Academy of Sleep Medicine (2007) The AASM manual for the scoring of sleep and associated events: rules, terminology and technical specifications. American Academy of Sleep Medicine, Westchester

2. Moser D, Kloesch G, Fischmeister FP, Bauer H, Zeitlhofer J (2010) Cyclic alternating pattern and sleep quality in healthy subjects - is there a first-night effect on different approaches of sleep quality? Biol Psychol 83(1):20-26

3. Toussaint M, Luthringer R, Schaltenbrand N, Nicolas A, Jacqmin A, Carelli G, Gresser J, Muzet A, Macher JP (1997) Changes in EEG power density during sleep laboratory adaptation. Sleep 20 (12):1201-1207

4. Senny F, Maury G, Cambron L, Leroux A, Destine J, Poirrier R (2012) The sleep/wake state scoring from mandible movement signal. Sleep Breath 16(2):535-542
5. Montgomery-Downs HE, Insana SP, Bond JA (2012) Movement toward a novel activity monitoring device. Sleep Breath. doi:10.1007/s11325-011-0585-y

6. Chung F, Liao P, Sun YM, Amirshahi B, Fazel H, Shapiro CM, Elsaid H (2011) Perioperative practical experiences in using a level 2 portable polysomnography. Sleep Breath 15(3):367-375

7. Hurley DA, Van de Water ATM, Holmes A (2011) Objective measurements of sleep for non-laboratory settings as alternatives to polysomnography - a systematic review. J Sleep Res 20 (1):183-200

8. Collop NA (2008) Portable monitoring for the diagnosis of obstructive sleep apnea. Curr Opin Pulm Med 14(6):525-529

9. Weir ID, Ahmed KM, Korbuly S, Achaen A, O'Malley M, O'Malley E, Manfredi C, Wakefield DB, Fine JM, Winter SM (2012) Detection of postoperative sleep-disordered breathing using a portable monitoring device. Sleep Breath. doi:10.1007/s11325-0110590-1

10. Berthomier C, Drouot X, Herman-Stoica M, Berthomier P, Prado J, Bokar-Thire D, Benoit O, Mattout J, d'Ortho MP (2007) Automatic analysis of single-channel sleep EEG: validation in healthy individuals. Sleep 30(11):1587-1595

11. de Chazal P, Fox N, O'Hare E, Heneghan C, Zaffaroni A, Boyle P, Smith S, O'Connell C, McNicholas WT (2011) Sleep/wake measurement using a non-contact biomotion sensor. J Sleep Res 20 (2):356-366

12. Wright KP, Johnstone Y, Fabregas SE, Shambroom JR (2008) Evaluation of a portable dry sensor based automatic sleep monitoring system. Sleep 31:A337-A337

13. Shambroom JR, Fabregas SE, Johnstone J (2012) Validation of an automated wireless system to monitor sleep in healthy adults. J Sleep Res 21(2):221-230

14. Anderer P, Gruber G, Parapatics S, Woertz M, Miazhynskaia T, Klösch G, Saletu B, Zeitlhofer J, Barbanoj MJ, Danker-Hopfe H, Himanen SL, Kemp B, Penzel T, Grozinger M, Kunz D, Rappelsberger P, Schlogl A, Dorffner G (2005) An E-health solution for automatic sleep classification according to Rechtschaffen and Kales: validation study of the Somnolyzer $24 \times 7$ utilizing the siesta database. Neuropsychobiology 51(3):115-133

15. Anderer P, Moreau A, Woertz M, Ross M, Gruber G, Parapatics S, Loretz E, Heller E, Schmidt A, Boeck M, Moser D, Kloesch G, Saletu B, Saletu-Zyhlarz GM, Danker-Hopfe H, Zeitlhofer J, Dorffner G (2010) Computer-assisted sleep classification according to the standard of the American Academy of Sleep Medicine: validation study of the AASM version of the Somnolyzer $24 \times 7$. Neuropsychobiology 62(4):250-264

16. Edinger JD, Bonnet MH, Bootzin RR, Doghramji K, Dorsey CM, Espie CA, Jamieson AO, McCall WV, Morin CM, Stepanski EJ (2004) Derivation of research diagnostic criteria for insomnia: report of an American Academy of Sleep Medicine work group. Sleep 27(8):1567-1596

17. Jasper HH (1958) Report on the Committee on Methods of Clinical Examination in Electroencephalography. The ten-twenty electrode system of the International Federation. Electroen Clin Neuro 10:370-375

18. Landis JR, Koch GG (1977) Measurement of observer agreement for categorical data. Biometrics 33(1):159-174

19. Kushida CA, Chang A, Gadkary C, Guilleminault C, Carrillo O, Dement WC (2001) Comparison of actigraphic, polysomnographic, and subjective assessment of sleep parameters in sleepdisordered patients. Sleep medicine 2(5):389-396

20. Fernandez-Mendoza J, Calhoun SL, Bixler EO, Karataraki M, Liao D, Vela-Bueno A, Jose Ramos-Platon M, Sauder KA, Basta M, Vgontzas AN (2011) Sleep misperception and chronic insomnia in the general population: role of objective sleep duration and psychological profiles. Psychosom Med 73(1):8897 